\title{
Fortbildung
}

\section{Symposium über Strabologie und Neuroophthalmologie 22. bis 23. 10. 2004 in Salzburg}

\author{
Einen AugenBlick bitte. 40 Jahre Orthoptistinnenausbildung in Salzburg \\ C. Scharinger \\ Salzburg
}

Bisher wurden in der Akademie für den orthoptischen Dienst in Salzburg knapp hundert Maturantinnen in 20 Lehrgänge aufgenommen. Die Ausbildung erfolgt nach dem MTD-Gesetz und der -Ausbildungsverordnung. Die Absolventinnen besetzen Stellen in Salzburg und den angrenzenden Bundesländern.

1964 begann die Sehschule Salzburg unter der damaligen Leiterin Dr. E. Friemel und deren leitender Orthoptistin Frau J. Attwenger mit der Ausbildung von Orthoptistinnen. Die Ausbildung wurde von der DOG anerkannt, das Examen musste in Deutschland abgelegt werden. 1971 wurde nach langen Bemühungen der Sehschule Salzburg und der Salzburger Landesregierung der orthoptische Dienst in die gehobenen medizinisch-technischen Dienste eingereiht, somit der Beruf in Österreich staatlich anerkannt und offiziell um Bewilligung der medizinisch-technischen Schule für den orthoptischen Dienst angesucht. Mit dem MTD-Gesetz 1992 kamen große Änderungen: dreijährige Dauer, Umbenennung in Akademien, neue Leitungsstruktur, Diplomarbeit, neue

\section{Okuläre Myasthenie}

K. Falb und D. Wurm

Zusammenfassung. Die Myasthenie beruht auf einer Störung der neuromuskulären Erregungsübertragung. Die häufigste Form wird durch Antikörper gegen die Acetylcholinrezeptoren an der neuromuskulären Synapse im Rahmen einer Autoimmunerkrankung hervorgerufen. Dabei scheint der Thymus eine zentrale Rolle in der Autoimmunpathogenese zu spielen.

Besonders häufig betrifft die postsynaptische Reduktion der Acetylcholinrezeptoren die Muskelendplatten der Augenmuskeln.

Während des Verlaufs einer Myasthenie treten bis zu 90\% Augenbewegungsstörungen auf. Die okuläre Myasthenie ist meist das erste Symptom einer Erkrankung. In $80 \%$ kommt es innerhalb von 2 Jahren zu einer Generalisierung, eine spätere Generalisierung ist eher selten. Für die okuläre Myasthenie sind eine ein-, beidseitige oder auch alternierende Ptose, so-
Fächer und Ausbildungsziele (z. B. Neuroophthalmologie, Neurologie, Rehabilitation von zentralen Sehstörungen und im Low-Vision-Bereich, pädagogische und psychologische Kenntnisse). Diese wurden notwendig, um Schlüssel- und Basisqualifikationen zur Gewährleistung einer eigenständigen und verantwortungsbewussten Ausübung und Weiterentwicklung des Berufes zu erwerben. Die Akademie in Salzburg kann durch das Naheverhältnis zu der Sehschule auf erfahrene Strabologen und Orthoptistinnen als Lehrer und Praktikumsanleiter zurückgreifen. Sie bietet ein breites Spektrum in der Grundausbildung, ihren Schwerpunkt sieht sie in der Vermittlung von speziellem Wissen und Können über Augenbewegungsstörungen und deren Diagnostik. Die Zukunft wird für alle gehobenen medizinisch-technischen Berufe und die Hebammen auch sechssemestrige Fachhochschulstudienlehrgänge mit Baccalaureatsabschluss bringen. Offen ist, ob ein solcher für die Orthoptik in Salzburg angeboten werden wird. wie Doppelbilder durch die Beteiligung der äußeren Augenmuskeln mit wechselnder Ausprägung im Tagesverlauf typisch.

Die differentialdiagnostische Abklärung erfolgt, neben der klinischen Untersuchung durch den Ophthalmologen und Neurologen und der Erstellung eines Antikörperstatus, mittels Tensilon-Test, EMG, MR, Liquoruntersuchungen und Muskelbiopsie. Wichtig ist auch die Suche nach Begleiterkrankungen, wie chronische Infektionen, die zu einer Exazerbation der Myasthenie führen können.

An 2 Fallbeispielen einer vorwiegend okulären Myasthenie werden der klinische Verlauf der Erkrankung sowie die therapeutischen Möglichkeiten aufgezeigt.

Fall 1 (männlich, geb. 1957) zeigt eine vorbestehende linksseitige congenitale Obliquus-superior-Störung, die 